\title{
The Missing Population of Be+Black Hole X-Ray Binaries
}

\author{
Aleksander Sądowski ${ }^{1}$, J. Ziółkowski ${ }^{1}$, K. Belczyński ${ }^{2}$ \\ and T. Bulik ${ }^{3}$ \\ ${ }^{1}$ Copernicus Astronomical Center, ul. Bartycka 18, 00-716 Warsaw, Poland \\ email: jz@camk.edu.pl \\ ${ }^{2}$ Department of Astronomy, New Mexico State University, \\ Frenger Mall, Las Cruces, NM 88003, USA \\ email: kbelczyn@lanl.gov \\ ${ }^{3}$ Astronomical Observatory of Warsaw University, Al. Ujazdowskie 4, \\ 00-478 Warsaw, Poland \\ email: tb@astrouw.edu.pl
}

\begin{abstract}
At present, $117 \mathrm{Be} /$ neutron star (Be/NS) X-ray binaries (XRBs) are known in the Galaxy and the Magellanic Clouds, but not a single Be/black hole (Be/BH) binary was found so far. We carried out the calculations of stellar population synthesis to investigate the case of the apparently missing population of Be/BH XRBs. According to our calculations, the main reason of this disparity is the fact that within the orbital period range where Be XRBs are found ( $\sim 10$ to $\sim 300$ days), these systems are formed predominantly with a NS component. The systems with a $\mathrm{BH}$ component are formed predominantly with much longer orbital periods and they are not easy to detect.
\end{abstract}

Keywords. X-rays: binaries, (stars:) binaries: close, stars: evolution, stars: emission-line, Be

\section{Introduction}

The binary systems composed of a Be star and a neutron star (Be/NS type systems) form the most numerous class of X-ray binaries (XRBs) in our Galaxy. These systems consist of a NS orbiting a Be type star on a rather wide (orbital periods in the range of $\sim 10$ to $\sim 300$ days), frequently excentric, orbit. NS has a strong magnetic field and, in vast majority of cases, is observed as an X-ray pulsar (with the spin periods in the range of $34 \mathrm{~ms}$ to about $6000 \mathrm{~s}$ ). The Be component is deep inside its Roche lobe and the mass accretion on a NS is occuring through the interaction of a NS with the excretion disc around Be component.

At present, 117 Be/NS type XRBs are known in the Galaxy and the Magellanic Clouds (which is almost a half of the total number of the known NS XRBs). Other classes of XRBs are less numerous: we know 87 X-ray bursters (which are also NS XRBs) and 48 X-ray pulsars not associated with a Be type companion (which form still other classes of NS XRBs: 35 of these NSs are associated with a supergiant type companion and 13 with a low mass companion). In addition, we know 58 black hole candidate (BHC) systems (among them 24 confirmed BH systems). However, not a single BHC binary containing a Be type component (Be/BH binary) was found so far.

This disparity (117 Be/NS type systems out of 252 known NS XRBs vs. not a single $\mathrm{Be} / \mathrm{BH}$ type system among 58 known $\mathrm{BH} \mathrm{XRBs}$ ) called the attention of the researchers already for some time. Zhang et al. (2004) noted that, according to stellar population synthesis calculations by Podsiadlowski et al. (2003), BH binaries are formed predominantly with relatively short orbital periods $\left(P_{\text {orb }}<10\right.$ days $)$. If this is the case, then, according 
to Zhang et al., the excretion disc truncation mechanism (Artymowicz \& Lubow, 1994) might be so efficient, that the accretion rate is very low and the system remains dormant (and therefore invisible) for almost all the time. One should note, however, that Podsiadlowski et al. considered, essentially, BH systems with Roche lobe filling secondaries, which definitely is not the case of Be XRBs. Therefore, their results are not relevant for the case of $\mathrm{Be} / \mathrm{BH}$ XRBs.

We carried out the calculations of stellar population synthesis to investigate the case of the apparently missing population of Be/BH XRBs using the Star Track code described by Belczyński, Kalogera \& Bulik (2002) and Belczyński et al. (2008).

\section{Definition of a Be XRB}

The most characteristic observational property of Be stars distinguishing them from other B stars is the presence of excretion discs producing the characteristic emission lines. The underlying cause of the presence of this disc is, in turn, rapid rotation. In the context of XRBs, the presence of an excretion disc is crucial, because it permits the relatively efficient accretion on the compact companion, even in the case of large orbital separation. It is not clear how Be stars achieved their fast rotation (although different hypothesis like rapid rotation at birth or spin-up due to binary mass transfer are advanced see e.g. McSwain \& Gies, 2005). The fraction of Be stars among all B stars is similar for single stars and for those in binary systems (one quarter to one third).

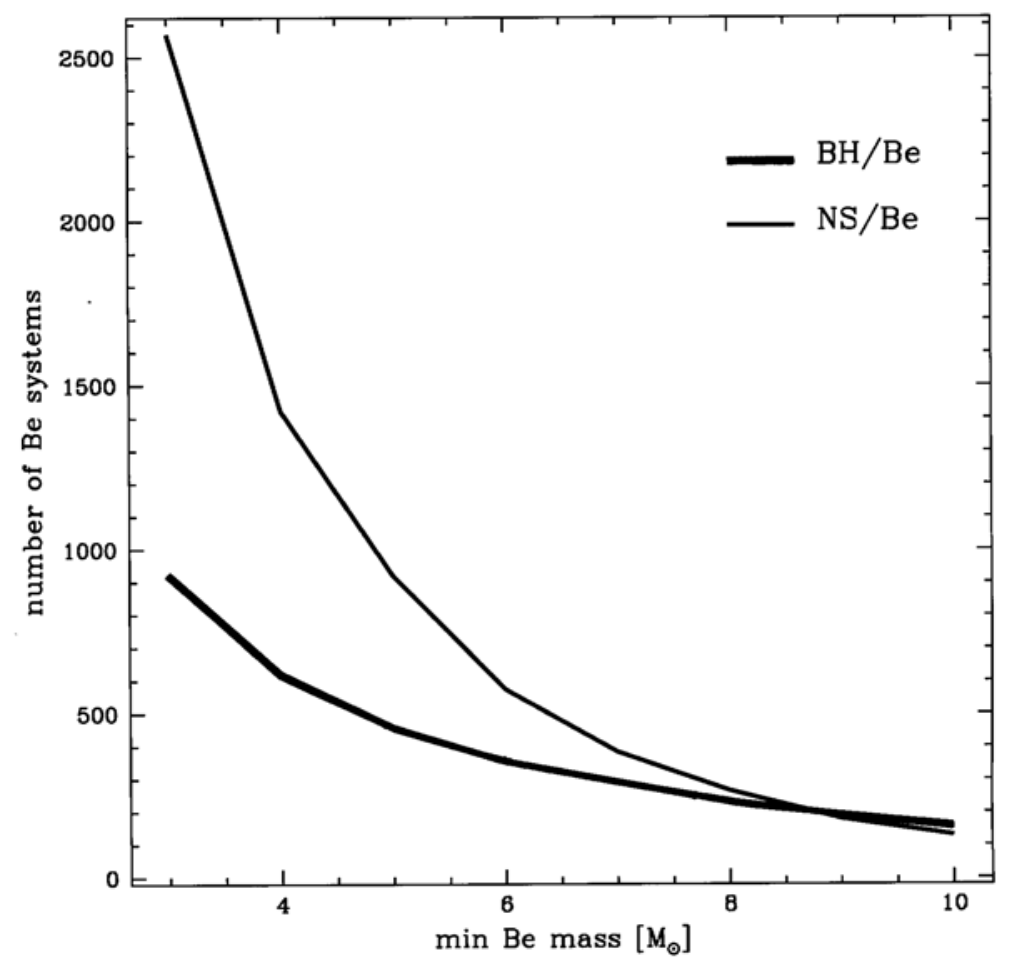

Figure 1. The expected numbers of $\mathrm{Be} / \mathrm{NS}$ (thin line) and $\mathrm{Be} / \mathrm{BH}$ (thick line) binaries as functions of the assumed minimal mass of a Be component. 
For the purpose of our calculations, we we assumed, for simplicity, that one quarter of all B stars are always Be stars and that these stars are always efficient mass donors, independently of the size of the binary orbit (as is, in fact, observed in Be/NS XRBs). Therefore, according to our definition, a Be XRB is a system composed of a compact object (NS or $\mathrm{BH}$ ) and a main sequence B star (and we apply a factor 0.25 to the number of such systems, to account for the fact that not every B star is a Be star).

\section{Preliminary Results}

Fig. 1 shows that, when we count the total expected numbers of $\mathrm{Be} / \mathrm{NS}$ and $\mathrm{Be} / \mathrm{BH}$ binaries, these numbers should be, roughly comparable. The estimated masses of observed Be stars cover the range from $\sim 2.3 \mathrm{M}_{\odot}$ (Lejeune \& Schaerer, 2001) to $\sim 25 \mathrm{M}_{\odot}(\mathrm{McSwain}$ $\&$ Gies, 2005). Therefore, if we assume $3 \mathrm{M}_{\odot}$ as a reasonable lower limit for the mass of a Be component, then the Be/NS systems should outnumber Be/BH systems only by a factor of about 2.5.

The reason for the observed large disparity becomes obvious, when we look at Fig. 2. According to our calculations, the distribution of the orbital periods is completely different for $\mathrm{Be} / \mathrm{NS}$ and $\mathrm{Be} / \mathrm{BH}$ systems. Within the orbital period range where $\mathrm{Be}$ XRBs are found ( 10 to $\sim 300$ days), Be systems are formed predominantly with a NS component. The ratio of the expected number of Be/NS systems to the expected number of $\mathrm{Be} / \mathrm{BH}$ systems is, for this orbital period range, larger than 50 . The systems with a $\mathrm{BH}$ component are formed predominantly with much longer orbital periods. Such systems are very difficult to detect, both due to very long orbital periods and due to, probably, very low luminosities (the accretion at such large orbital separations must be very inefficient).
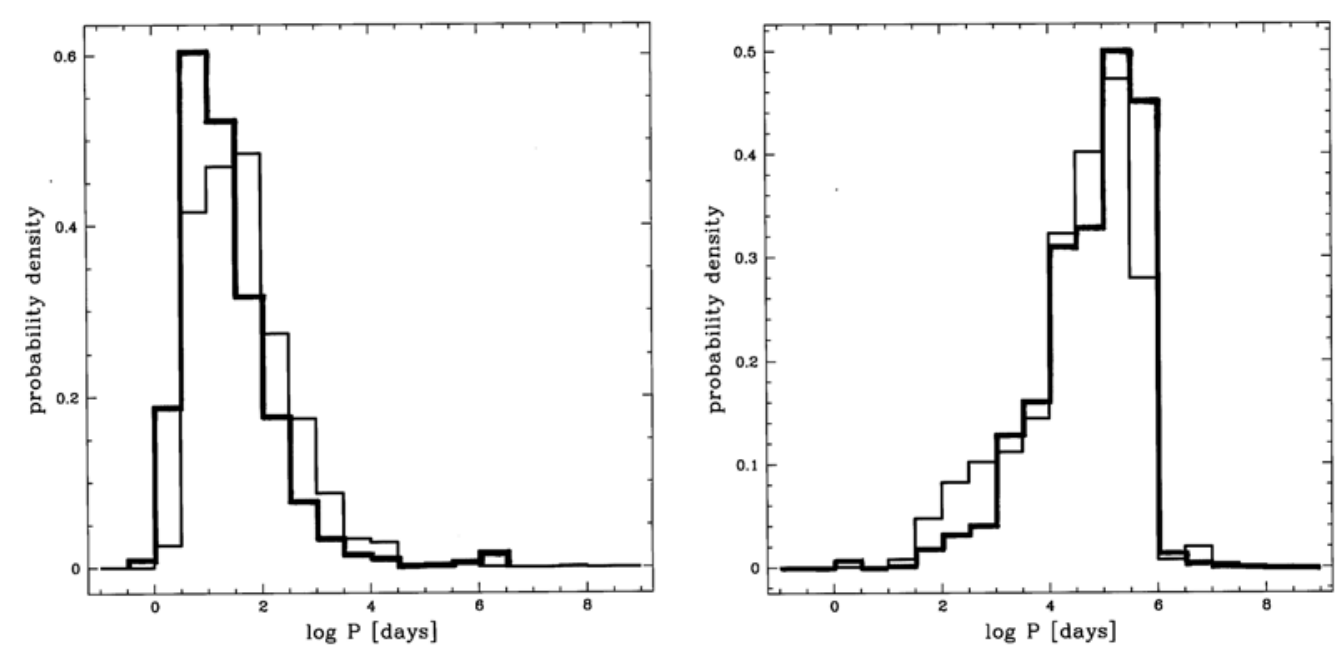

Figure 2. The expected distributions of orbital periods of Be/NS (left panel and $\mathrm{Be} / \mathrm{BH}$ (right panel) binary systems. Thick lines correspond to the minimum mass of a Be component equal $3 \mathrm{M}_{\odot}$ and the thin lines to the minimum mass equal $8 \mathrm{M}_{\odot}$.

We should stress, that the results presented above are only very preliminary results. We plan to carry out further calculations. In particular, we shall try to explain the physical 
reasons for so different orbital periods distributions for the systems with NSs and with BHs.

We should also stress that, while our results provide a major factor explaining the observed disparity in the numbers of $\mathrm{Be} / \mathrm{NS}$ and $\mathrm{Be} / \mathrm{BH}$ systems, this might be not the only factor. Another possible factor may be related to the previous evolution of a Be star. If, indeed, a B star must be a member of a binary system and undergo a mass transfer in order to become a Be star, then one can imagine that the systems composed of a Be star and a relatively less massive companion (which collapses to a NS) remain bound, while those composed of a Be star and a relatively more massive companion (which collapses to a $\mathrm{BH})$ are disrupted in the process of supernova explosion.

\section{Acknowledgements}

This work was partially supported by the polish MNiSW grant NN203065933 (20072010).

\section{References}

Artymowicz, P. \& Lubow, S. H. 1994, ApJ 421, 651

Belczyński, K., Kalogera, V., \& Bulik, T. 2002, ApJ 572, 407

Belczyński, K., Kalogera, V., Rasio, F.A., Taam, R.E., Zezas, A., Bulik, T., Maccarone, T.J., \& Ivanova, N. 2008, ApJ Suppl (in press), also astro-ph/051181

Lejeune, T. \& Schaerer, D. 2001, A\&BA 366, 538

McSwain, M.V. \& Gies, D.R. 2005, ApJ Suppl 161, 118

Podsiadlowski, Ph., Rappaport, S., \& Han, Z. 2003, MNRAS 341, 385

Zhang, F., Li, X.-D., \& Wang, Z.-R. 2004, ApJ 603, 663

\section{Discussion}

Podsiadlowski: I did not quite understand the evolution that produces a black hole with a $\sim 3 \mathrm{M}_{\odot}$ companion and an orbital period of 400 days. Such systems should have experienced a common envelope phase.

BELCZYŃSKI: Indeed such systems experience a common envelope (CE) phase. Progenitor system consists of a massive star $\left(\sim 40 \mathrm{M}_{\odot}\right)$ and a companion may have several solar masses. A system is initially on a very wide orbit $\left(2000-4000 \mathrm{R}_{\odot}\right)$. When the primary evolves two important (for the CE survival) things are happening: (i) core of the massive primary grows in mass (nuclear burning). (ii) mass of the primary envelope decreases in mass (wind mass loss). If the orbit is wide enough (as it is for progenitors of BHs with low mass companions) the primary fills its Roche lobe when substantial part of its envelope is gone. The following CE phase tightens the orbit by factors 10-100. Usually, it is expected that such a CE phase leads to a merger of two stars, since it is argued that there is not enough binary orbital energy in the system (due to the very low mass of the companion star) to unbind the massive envelope of the primary. However, in some cases enough of the envelope is lost that the orbital decay during the CE phase does not lead to a merger. The exposed helium core of the primary follows through subsequent evolution and forms a black hole. The massive black hole with low mass companion system is formed. Such a scenario requires that the stellar winds of massive stars are lowered by factor of about 2 (so that the wind mass loss from the massive primary does not prevent Roche lobe overflow and the following CE phase), the assumption that is well within the observational mass loss estimates available for massive stars. The example of such an evolution is given in full detail in Belczynski \& Bulik 2002, ApJ Lett. 574, L147. The presented example was used to explain self-consistently the formation of microquasar 
GRS 1915+105, a binary system hosting the most massive known stellar black hole in our Galaxy.

LANGER: Which physics principle explains the key feature in your analysis, namely that $\mathrm{BH}$ binaries have much larger periods than NS binaries?

BELCZYŃSKI: In general, the black hole binaries originate from the (initially) wider systems for a simple reason that stars that form black holes are more massive (and therefore larger in size) than neutron star progenitors. To avoid the merger during one of the subsequent Roche lobe overflow phases the binary systems that form black holes with companions (and not the mergers) are wider. However, for the specific subpopulations of binary systems discussed in our paper we are still investigating the detailed evolutionary channels and we will present the detailed analysis and the full answer to this question in our journal submission. 